\title{
Evolution of Structural-Phase States in TiNi Surface Layers Synthesized by Electron Beam Treatment
}

\author{
L. L. Meisner, A. I. Lotkov, Yu. P. Mironov, and A. A. Neyman \\ Institute of Strength Physics and Materials Science, Siberian Branch of the Russian Academy of Sciences, Tomsk 634021, Russia
}

Correspondence should be addressed to L. L. Meisner, meisner21@yahoo.com

Received 1 October 2009; Accepted 10 January 2010

Academic Editor: Abdel Salam H. Makhlouf

Copyright () 2010 L. L. Meisner et al. This is an open access article distributed under the Creative Commons Attribution License, which permits unrestricted use, distribution, and reproduction in any medium, provided the original work is properly cited.

The paper presents the results of X-ray diffraction analysis of nonequilibrium structural and elastic stress states in TiNi surface layers irradiated by low-energy electron beams. It is found that a surface layer with a mixed (2D columnar and 3D equiaxial) submicrocrystalline structure is formed on the irradiated side of the TiNi specimens, and the volume fractions of the two structure types depend on the beam energy parameters and number of pulses. The B2 phase synthesized in the layer is characterized by lattice microstrain due to stresses of the first and second kinds $\left(\varepsilon^{\mathrm{I}} \approx \pm 1 \%, \varepsilon^{\mathrm{II}}=0.25 \%\right)$, and the layer as such is an internal stress concentrator for underlying layers of the material. In the intermediate layer beneath the stress concentrator, relaxation of irradiation-induced internal stress takes place. It is shown that the main mechanism of the relaxation is partial B2 $\rightarrow$ B19' martensite transformation. The $\mathrm{B} 19^{\prime}$ martensite phase in the intermediate layer decreases the microstrain in the conjugate $\mathrm{B} 2$ phase. The thickness of the layer in which the relaxation processes develop through the B2 $\rightarrow$ B19' martensite transformation is $10-15 \mu \mathrm{m}$.

\section{Introduction}

Recently, there has been intensive development in surface modification of metals by a high current electron beams of moderate and low energy [1-8]. The treatment makes it possible to form surface layers with new physical properties while unaffecting the original features in the material bulk. Crystalline and amorphous states in the layers, as a rule, are metastable since the temperature, pressure, heating and cooling conditions under which they are formed are highly nonequilibrium [7-13]. Available experimental data show that it is these nonequilibrium states in surface layers that ensure new atypical properties attractive for practical applications [14-19]. It is apparent that the new properties are primarily due to the changes in structural-phase states of material and hence in properties of its surface layers on electron beam treatment. However, the information on the thus synthesized structural-phase states and their relation to the change in surface properties of materials is very scanty, and the mechanisms of their formation are poorly known.

Electron beam treatment of material gives rise to strong internal stress fields localized in its near-surface volumes
$[7,10,20-23]$. In the regions of stress field localization, the material undergoes a change in mechanical properties (an increase in hardness, brittleness, rigidity) $[18,24]$; however, there is also evidence that the stress fields adversely affect the chemical properties of the treated material, for example, impair its corrosion resistance $[17,19,25]$.

TiNi-based alloys with shape memory effect display elastic properties similar to those of biological tissues and this permits their advantageous use in medicine. However, the highly toxic nickel present in large concentration in TiNi-based alloys and the risk of its release into a biological medium makes urgent the search for ways of depositing protective coatings on their surface to increase the corrosion resistance and hence biocompatibility of the alloys. Experiments show that a promising technique for attaining the goal is low-energy electron beam treatment $[15,17,19]$. It is found that this treatment results in nonequilibrium structural-phase states in near-surface regions $[17,19,26]$ and the gradients of their structural parameters provide a considerable increase in the physicochemical and mechanical characteristics of the alloys. 
At the same time, it should be noted that despite the large body of research in these structural states, no unified concept has been developed so far to combine mechanisms of their formation and methods of parameter control (grain size, phase composition, atomic structure parameters). This is primarily due to uniqueness of the object under study, since even with one and the same material and processing technique the process parameters vary from one experiment to another requiring a separate examination in each particular case.

The objective of the work is to study the mechanisms of the formation and evolution of nonequilibrium structural and elastic stress states in TiNi near-surface layers irradiated by low-energy electron beams.

\section{Materials, Method of Surface Treatment, and Investigation Technique}

The TiNi alloy to be examined was produced by electric arc melting of iodide titanium and $\mathrm{HO}$ nickel with sixfold remelting of the ingot. The initial components of the mixture-titanium and nickel-were taken in the ratio corresponding to the equiatomic one (50 at.\% $\mathrm{Ti}+50$ at.\% $\mathrm{Ni})$. In the work, we controlled the chemical composition in surface layers by Auger layer-by-layer electron spectroscopy, $\mathrm{X}$-ray diffraction analysis and X-ray spectrum analysis. It was found [18] that selective evaporation of nickel escaped detection and after remelting, the composition of the B2 phase in the alloy corresponded to 49.5 at. $\% \mathrm{Ti}+50.5$ at.\% $\mathrm{Ni}$, that is, $\mathrm{Ti}_{49.5} \mathrm{Ni}_{50.5}$.

After electroerosion cutting to dimensions of $20 \times 10 \times$ $1 \mathrm{~mm}^{3}$, finishing heat treatment (annealing at $\mathrm{T}=1073 \mathrm{~K}$ for $1 \mathrm{~h}$ with cooling in a furnace) and electropolishing, the TiNi alloy specimens (further-TiNi specimens) at room temperature were in the two-phase state: main phase with B2 structure (bcc lattice ordered as $\mathrm{CsCl}$, start temperature of the $\mathrm{B} 2 \rightarrow \mathrm{B} 19^{\prime}$ transformation $\mathrm{M}_{\mathrm{S}} \approx 283 \mathrm{~K}, \mathrm{~B} 2$ lattice parameter for the $\mathrm{Ti}_{49.5} \mathrm{Ni}_{50.5}$ composition $a_{0 \mathrm{~B} 2} \approx$ $0.30125 \pm 0.00005 \mathrm{~nm})$ and small amount $(<5 \% \mathrm{vol})$ of the intermetallic $\mathrm{Ti}_{2} \mathrm{Ni}$ phase.

Pulsed electron beam treatment was realized at the Institute of High Current Electronics, SB RAS (Tomsk). The TiNi specimen surface was irradiated by a low-energy (up to $30 \mathrm{keV}$ ) high-current (up to $30 \mathrm{kA}$ ) electron beam in high vacuum $\left(\sim 10^{-6} \mathrm{~Pa}\right)$ with oilless pump-down in the surface melting regime. The pulse duration was $2.6 \div 3.4 \mu$ s. With a beam energy density $E_{1}=4 \mathrm{~J} / \mathrm{cm}^{2}$ and $E_{2}=8 \mathrm{~J} / \mathrm{cm}^{2}$, the specimens were irradiated by twelve and fifty pulses, respectively.

The structural-phase states in the alloy after electron beam treatment are highly nonequilibrium and hence the entire complex of X-ray diffraction analysis, including the variation in the $\mathrm{X}$-ray diffraction geometry, was repeated one year later. X-ray diffraction analysis of the structuralphase states in near-surface regions of the TiNi specimens was made on a DRON-7 diffractometer at room temperature immediately after the treatment and one year later. In the analysis, the symmetric and asymmetric (variable grazing

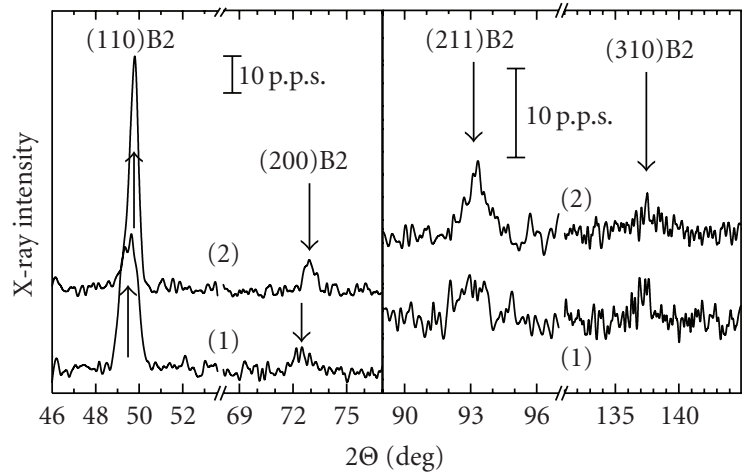

FIgURE 1: Fragments of X-ray diffraction patterns for the TiNi specimens exposed to electron beam surface treatment with $E_{1}=$ $4 \mathrm{~J} / \mathrm{cm}^{2}$, Co- $\mathrm{K}_{\alpha}$ radiation, asymmetric diffraction scheme: $\alpha=$ $0.15^{\circ}(1)$ and $\alpha=2^{\circ}(2)$.

angle $\alpha$ ) Bragg diffraction geometry with $\mathrm{Co}-\mathrm{K}_{\alpha}$ and $\mathrm{Cu}-\mathrm{K}_{\alpha}$ wavelengths were used. In the asymmetric X-ray reflection scheme, a constant effective penetration depth $h$ at all Bragg angles was attained by choosing the angle $\alpha$ according to the procedure described in [27] and the instrumental shift of diffraction peaks was minimized by accurate adjustment.

In the asymmetric Bragg diffraction geometry, the angle $\alpha$ was decreased to $\alpha=0.1^{\circ}$ (without rotating the specimen), that is, to an angle smaller than the so-called critical angles of total external reflection $\alpha_{c}$ with the chosen X-ray wavelengths for titanium nickelide (for both types of radiation $0.3^{\circ}<$ $\left.\alpha_{\mathrm{TiNi}}<0.4^{\circ}\right)$. Nevertheless, a clearly defined diffraction pattern of four to five B2 peaks with a halfwidth of $\sim 1^{\circ}$ was observed even at the so small glancing angle. Similar peaks are shown in Figure 1. Evidently, the diffraction pattern observed at angles smaller than the theoretical angle of total external reflection for the B2 phase owes to nonzero surface roughness of the test specimens. For reducing the instrumental broadening of X-ray profiles, we used narrow $(0.05 \mathrm{~mm})$ exist slits and limited the specimen width. This allowed estimation of the microstrain in a layer of thickness up to $\sim 50 \mathrm{~nm}$ (on the assumption that the height of irregularities is smaller than or comparable with this value).

The microstrain $\varepsilon^{\mathrm{I}} \equiv \varepsilon_{\psi}$ (due to the stress of the first kind) of the B2 phase lattice in near-surface regions was determined by measuring the angular positions of peaks of the $\mathrm{B} 2$ structure in the irradiated and nonirradiated specimens and by calculating the lattice parameter of the B2 phase from each peak and then from the formula [28]

$$
\varepsilon_{\psi}=\frac{a_{\psi}-a_{0}}{a_{0}},
$$

where $\Psi=\left(\Theta_{\mathrm{hkl}}-\alpha\right)$ and is the angle between the normal to the specimen surface and the normal to the reflection plane (hkl), $\Theta_{\mathrm{hkl}}$ is the Bragg angle, $\alpha$ is the angle between the direction of the primary beam and the surface plane (glancing angle). The microstrain of the $\mathrm{B} 2$ phase lattice was divided into normal and tangential (to the surface plane) components using the extrapolation curves $a\left(\sin ^{2} \Psi\right)$ 


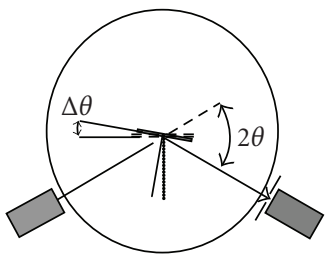

(a)

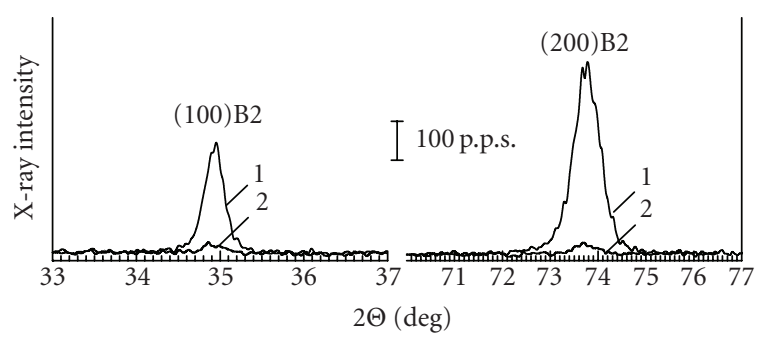

(b)

FIGURE 2: Misorientation of the specimen position by the angle $\Delta \theta$ relative to the position in the symmetric Bragg diffraction geometry (a), fragments of X-ray diffraction patterns for the TiNi specimens irradiated with $E_{1}=4 \mathrm{~J} / \mathrm{cm}^{2}$ (b): Bragg diffraction geometry (1), Bragg diffraction geometry with misorientation of the specimen position (scheme a) by the angle $\Delta \theta=1.5^{\circ}(2)$. Co- $\mathrm{K}_{\alpha}$ radiation, entrance and exit horizontal slits of width $0.1 \mathrm{~mm}$.

and the values of $a_{\psi}$ determined from the curves ( $\Psi=0^{\circ}$ corresponds to the lattice parameter of the $\mathrm{B} 2$ phase and hence to the lattice microstrain normal to the plane of the specimen surface, and $\Psi=90^{\circ}$ to that tangential to this plane). The measurement accuracy $\Delta \varepsilon_{\psi}= \pm 0.0005$.

The size of coherent scattering regions $D$ was estimated using an approximation technique with separation of the contributions of the microstrain $\varepsilon^{\mathrm{II}}$ (due to the stress of the second kind) and crystallite size to diffraction line broadening. The measurement accuracy for the microstrain $\Delta \varepsilon^{\mathrm{II}}= \pm 0.0005$, and for coherent scattering regions of $50 \div$ $10 \mathrm{~nm}, \Delta D$ varies within $\pm 12 \div 1 \mathrm{~nm}$.

The structure of the irradiated surface was examined in reflected electrons using an Scanning electron microscope LEO EVO 50 (Zeiss, Germany) and in backscattered electrons using an INCA Crystal and EBDS systems (Oxford Instruments).

\section{Results and Discussion}

The X-ray diffraction analysis performed immediately after electron beam irradiation [27] shows that on the irradiated side of the TiNi specimens, a surface (outer) layer with atomic structure parameters differing from those in the nonirradiated TiNi specimens is normally formed. This layer is characterized by a one-phase structural state in which the lattice parameter of the B2 phase is smaller than that before irradiation and by texture parameters different from those in the nonirradiated specimens; this is evidenced from intense superstructure $(100)_{\mathrm{B} 2}$ and main $(200)_{\mathrm{B} 2}$ peaks present on the X-ray diffraction patterns of the treated specimens and absent on those of the initial specimens.

The X-ray diffraction analysis performed within a year after the irradiation and presented in the work show that with misorientation of the specimen by a certain angle $\Delta \theta$ relative to the position in the symmetric Bragg diffraction geometry (Figure 2(a)), the intensity of the $(100)_{\mathrm{B} 2}$ and $(200)_{\mathrm{B} 2}$ peaks decreases steeply and at $\Delta \theta \geq 2^{\circ}$ they disappear (Figure 2(b), 2). Unlike the above peaks, $B 2$ peaks with other indices (hkl) survive on the $\mathrm{X}$-ray patterns and feature an asymmetric profile (Figure 3(a)) with noticeable smearing (Figure 3(b)) compared to the initial shape before irradiation.

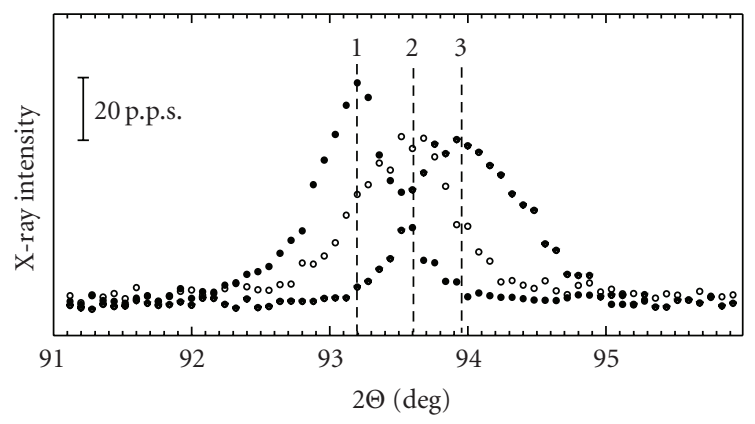

(a)

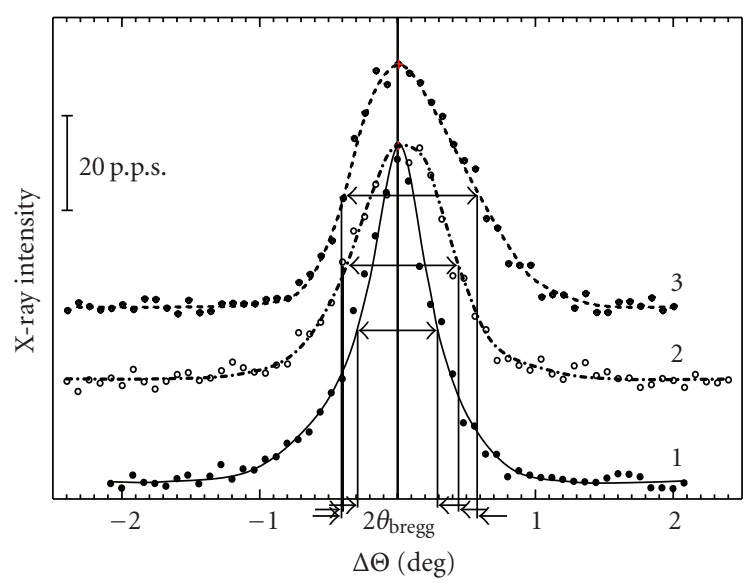

(b)

FIGURE 3: Position (a) and shape (b) of the (211) B2 peak in the TiNi specimen: before irradiation (1), after irradiation with $E_{1}=$ $4 \mathrm{~J} / \mathrm{cm}^{2}, N=20$ pulses (2) and with $E_{2}=8 \mathrm{~J} / \mathrm{cm}^{2}, N=50$ pulses (3). The Bragg diffraction geometry, $\mathrm{Co}-\mathrm{K}_{\alpha}$ radiation, entrance and exit horizontal slits of width $0.1 \mathrm{~mm}$.

Apparently, the foregoing effects are impossible to explain by the presence of the phase with a purely columnar structure in the layer formed after irradiation. According to the X-ray data, the phase state in the layer is characterized by the $\mathrm{B} 2$ structure with a lattice parameter smaller than that in the initial B2 structure; thus, it can be supposed that there are two microstructural modifications of the 


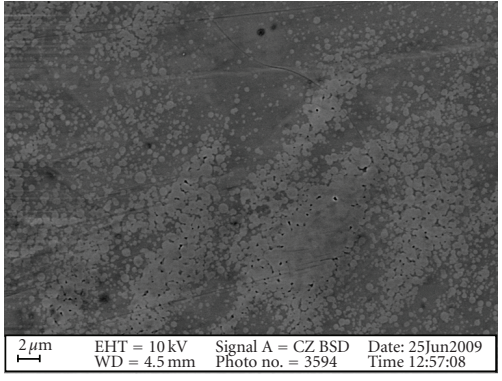

(a)

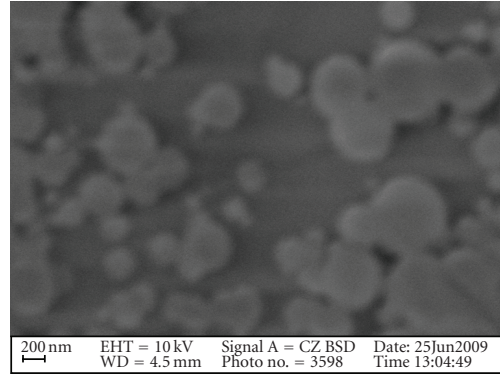

(b)

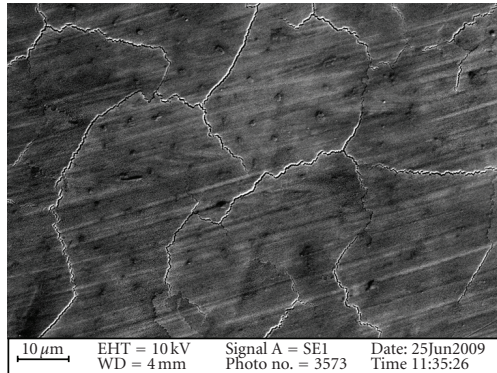

(c)

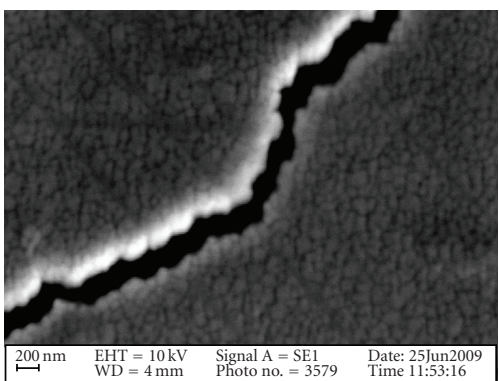

(d)

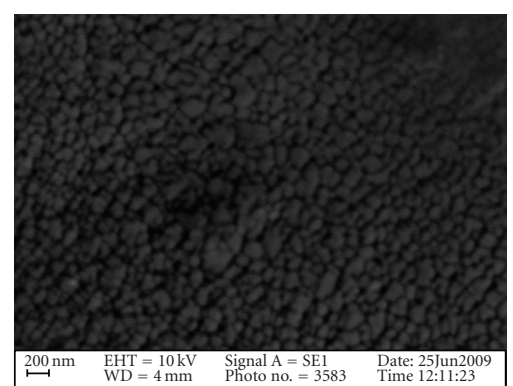

(e)

FIGURE 4: SEM images of the TiNi specimen surface after electron beam irradiation: with $E_{1}=4 \mathrm{~J} / \mathrm{cm}^{2}, N=20$ pulses in backscattered electrons (a), (b) and with $E_{2}=8 \mathrm{~J} / \mathrm{cm}^{2}, N=50$ pulses in secondary electrons (c)-(e).

synthesized B2 phase. The first modification is a columnar structure consisting of single crystallites with coherent scattering regions of size $>100 \mathrm{~nm}$ in a direction normal to the specimen surface, that is, their size is much larger than the linear dimensions of the base of an individual crystallite. Estimation of the sizes of coherent scattering regions from the $(100)_{\mathrm{B} 2}$ and $(200)_{\mathrm{B} 2}$ peaks for different misorientations of the specimens shows that they remain near-constant. Therefore, this type of structural modification can be identified as a $2 \mathrm{D}$ submicrocrystalline state (by analogy with the Gleiter classification of nanostructures [29]).

The second microstructural modification of the B2 phase more closely corresponds to a $3 \mathrm{D}$ submicrocrystalline structure with coherent scattering regions of size $60 \div$ $70 \mathrm{~nm}$. The size of the regions calculated from B2 peaks with different indices $(\mathrm{hkl})_{\mathrm{B} 2}$ is no greater and sometimes even 1.5 times smaller $(\sim 40 \mathrm{~nm})$ than the above values. However, the layers contributed to the diffraction peaks with different indices (hkl) differ in thickness and it is impossible to take into complete account the layer thickness. Thus, the data on coherent scattering regions of small size $(\sim 40 \mathrm{~nm})$ are qualitative suggesting that there is no coherent scattering region larger than $60 \div 70 \mathrm{~nm}$ in the layer under consideration.

The surface morphology of the specimens subjected to different modes of electron beam irradiation was studied in secondary and backscattered electrons by scanning electron microscopy methods. The study reveals structures consisting of submicrocrystalline grains that fill in whole (Figures $4(\mathrm{c})-4(\mathrm{e})$ ) or in part (Figures $4(\mathrm{a})$ and $4(\mathrm{~b})$ ) the specimen surface. It is seen in Figure 4(c) that the specimens irradiated by 50 pulses with the highest energy are cracked after irradiation. The surface cracks apparently develop along grain boundaries of the initial $\mathrm{B} 2$ phase, since they form a cellular structure with a cell size of $25 \div 30 \mu$ m coincident with the grain size in the structure before irradiation. However, inside the cells there is a finer submicrocrystalline grain structure with a grain size of $20 \div 200 \mathrm{~nm}$, and the step shape of the cracks is indicative of cracking along the grain boundaries of the structure. Increasing the electron beam energy deposited to the surface layer causes an increase in tensile stress (parallel to the specimen plane) and thus fracture of the surface layer as the ultimate strength of the material is reached. However, as is shown in the work and consistent with the mechanisms proposed in [22], the residual tensile stress decreases to almost zero even at a depth of $2-5 \mu \mathrm{m}$. It is this fact that is responsible for cracks only at the specimen surface and their non-propagation deep into the material. Thus, examination by scanning electron microscopy supports the results of X-ray diffraction analysis and suggests that a submicrocrystalline layer with a $2 \mathrm{D}$ (2D substructure) or 3D (3D substructure) type of grain morphology can be formed in the surface layer of the TiNi specimens on electron beam treatment.

Estimation of the elastic stress state in the layer of the synthesized B2 structure reveals high lattice microstrain $\varepsilon^{\mathrm{I}} \approx$ $\pm 1 \%$ due to stress of the first kind and rather low microstrain $\mathcal{\varepsilon}^{\mathrm{II}}=0.25 \%$ due to stress of the second kind. The stress of the first kind presumably arises in response to changes in the external conditions of electron beam treatment (propagation of the temperature front and elastic wave, cooling rate, 


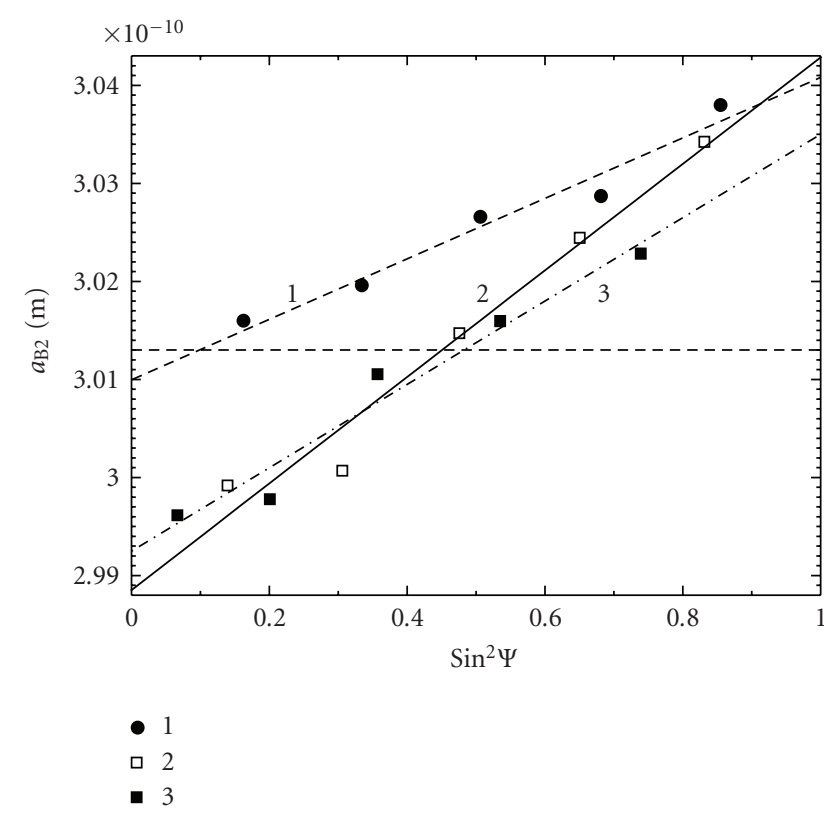

FIgURE 5: Lattice parameter $a_{B 2}$ versus $\sin ^{2} \psi$ in the TiNi specimens after irradiation with 20 pulses at $E_{1}=4 \mathrm{~J} / \mathrm{cm}^{2}: \alpha=1^{\circ}(1), \alpha=3^{\circ}(2)$ and $\alpha=10^{\circ}(3) ; a_{0}$ is the lattice parameter of the B2 phase in the initial specimen.

duration; number of pulses, etc.) and levels off throughout the specimen bulk. The stress of the second kind results from dispersion of "blocks" (or coherent scattering regions) produced on irradiation and from microdistortions in the blocks.

Evidently, the elastically stressed outer layer is a stress concentrator for underlying TiNi layers, that is, for intermediate layers between the synthesized outer layer and the unaffected material bulk, and is bound to influence the structural states of the B2 phase in these layers. Actually, $\mathrm{X}$-ray diffraction analysis of the specimens exposed to lowenergy electron beams shows that in the layers beneath the outer layer, the lattice parameter of the B2 phase varies with both the thickness of the analyzed layer and the mutual orientation of the primary beam and of the plane of the specimen surface, that is, with crystallographic direction.

It is evident from the dependences of the parameter $a_{\mathrm{B} 2}$ on the function $\sin ^{2} \Psi$ (Figure 5) for different glancing angles that after irradiation a linear increase in $a_{\mathrm{B} 2}$ with an increase in $\sin ^{2} \Psi$ occurs in the TiNi specimens. The slope angles of the straight lines $a_{\mathrm{B} 2}\left(\sin ^{2} \Psi\right)$ depend on the glancing angle $\alpha$; however at any $\alpha$, these lines have a positive slope such that $a_{\mathrm{B} 2}(\Psi=0)<\left(a_{\mathrm{B} 2}\right)<a_{\mathrm{B} 2}\left(\Psi=90^{\circ}\right)$. Besides, the dependences $a_{\mathrm{B} 2}\left(\sin ^{2} \Psi\right)$ in Figure 5 are indicative of the gradient of the lattice parameter in the synthesized B2 structure; the smallest values of the gradient are found near the irradiated surface. The most significant change in $a_{\mathrm{B} 2}$ is revealed in a surface layer of thickness less than $2 \mu \mathrm{m}$ to which there corresponds $\alpha=3^{\circ}$ (straight line 2 in Figure 5). Obviously this behavior of $a_{\mathrm{B} 2}$ is due to the elastic stress fields localized in near-surface layers exposed to electron beam treatment.
The slope of the straight lines $a_{\mathrm{B} 2}\left(\sin ^{2} \Psi\right)$ makes it possible to estimate the orientation dependence and the type (compressive/tensile) of the elastic stress state in the phase under study [20]. In the example, electron beam irradiation gives rise to compressive stress normal to the surface plane and tensile stress tangential to it in a surface layer of thickness up to $10 \div 15 \mu \mathrm{m}$ (the Co- $\mathrm{K}_{\alpha}$ penetration depth is $\sim 1.3$ and $\sim 3.8 \mu \mathrm{m}$ for $\alpha=3^{\circ}$ and $10^{\circ}$, resp.). At the same time, the values of $a_{\mathrm{B} 2}$ measured at an angle $\Psi=45^{\circ}$ are close to those in the initial specimen, that is, the average value of the lattice parameter of the B2 phase in the elastically stressed layer remains the same, and hence the concentration relation between the $\mathrm{Ti}$ and $\mathrm{Ni}$ components in this layer remains constant.

Estimation of the microstrain $\varepsilon^{I} \equiv \varepsilon_{\psi}$ in the lattice of the B2 phase immediately after electron beam treatment shows that in an outer layer of thickness up to $1 \div 3 \mu \mathrm{m}$ the microstrain takes on the highest values and decreases with increasing the penetration depth. The microstrain $\varepsilon_{\Psi}$ calculated from the X-ray diffraction patterns within a year after irradiation is suggestive of a relaxation process in a layer of thickness less than $1 \mu \mathrm{m}$, as evidenced also by a change in the character of the penetration depth dependence of microstress. Figure 6 shows the microstrain $\varepsilon_{\Psi}$ in relation to the penetration depth for mutually perpendicular directions corresponding to $\Psi=0^{\circ}$ and $\Psi=90^{\circ}$. It is seen from the figure that immediately after irradiation, the tangential component of the microstrain $\varepsilon_{\psi=90^{\circ}}$ is positive over the entire range of measurements (curve 1) and this gives an indication of tensile internal stress in this direction. The highest values of $\varepsilon_{\psi}=90^{\circ}$ are attained in a layer of thickness less than $2 \mu \mathrm{m}$. Within a year after irradiation, this dependence remains the same (curve 2).

Unlike the tangential component, the normal component of the microstrain $\varepsilon_{\psi=0}$ in the surface layer is negative (curve 3) and this points to compressive internal stress in this direction. Immediately after irradiation, the normal component of the microstrain reaches the highest (absolute) values in a layer of thickness less than $1 \mu \mathrm{m}$ and one year later its absolute values in the modified layer decrease and fluctuate about zero (curve 4). At a depth of $1 \mu \mathrm{m}$ from the surface, the values of $\varepsilon_{\psi=0}$ obtained immediately and within a year after irradiation are near coincident. This means that no elastic stress relaxation occurs at this depth. A small discrepancy in $\varepsilon_{\psi=0}$ found for a thickness of $1 \div 4 \mu \mathrm{m}$ one year later is likely to be due to the change in the microstrain of the B2 phase lattice in the recrystallized layer, which contributes to the X-ray diffraction patterns.

As already noted the alloy under study experiences B2 $\leftrightarrow$ B19' martensite transformation whose temperatures are close to room temperature $\left(T_{r}\right)$. First, this means that with the advent of internal elastic stress fields in the specimens, the stress-induced B2 $\rightarrow$ B19' transformation may result in the B19' martensite phase; second, the B19' phase may appear on X-ray patterns taken at room temperature rather than in temperature chambers.

Actually in the range of angles $2 \theta \approx 38 \div 44^{\circ}$ (for $\mathrm{Cu}-\mathrm{K}_{\alpha}$ radiation), the X-ray diffraction patterns taken in the asymmetric Bragg geometry reveal rather clearly defined 


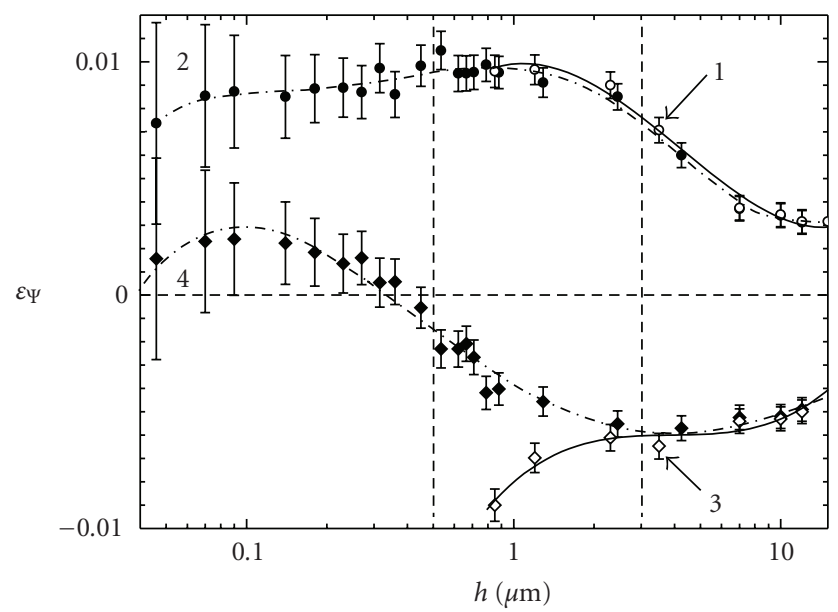

Figure 6: Components of the microstrain in the B2 phase lattice in relation to the penetration depth in the TiNi specimens irradiated with $E_{1}=4 \mathrm{~J} / \mathrm{cm}^{2}: \varepsilon_{\Psi=90^{\circ}}=90(1,2)$ and $\varepsilon_{\Psi=0}(3,4)$. Curves $1(\circ)$, $3(\diamond)$-immediately after irradiations, curves $2(\bullet), 4(\diamond)$-a year later.

B19' peaks whose intensity varies with the angle $\alpha$. To the above range of angles there corresponds a penetration depth of 6 and $7 \mu \mathrm{m}$ (for $\mathrm{Co}-\mathrm{K}_{\alpha}$ and $\mathrm{Cu}-\mathrm{K}_{\alpha}$ radiation, resp.) and this implies that the $\mathrm{B} 19^{\prime}$ martensite phase is formed in the layer beneath the outer layer (with submicrocrystalline structures). Figure 7 shows the ratio of the total intensity of all $\mathrm{B} 19^{\prime}$ peaks revealed on the X-ray diffraction pattern to the intensity of the $(110)_{\mathrm{B} 2}$ peak characteristic of the volume fractions of the martensite and high-temperature phases $\left(v_{\mathrm{B} 19^{\prime}} / v_{\mathrm{B} 2}\right)$ throughout the layer contributing to the diffraction pattern. It follows from the dependence that the volume fraction of the martensite phase near the surface is minimum, varies in a surface volume with a maximum at a depth of $4-6 \mu \mathrm{m}$ from the irradiated surface, and tends to decrease in deeper layers.

Comparison of the dependences in Figures 6 and 7 shows that there is a correlation in the change of the microstrain $\varepsilon_{\Psi}$ in the B2 phase lattice and the amount of the B19' martensite phase with increasing the thickness of the analyzed layer. At a depth of 3-6 $\mu \mathrm{m}$ from the specimen surface, the both components of $\varepsilon_{\Psi}$ take on the smallest (absolute) values and $v_{\mathrm{B} 19^{\prime}} / v_{\mathrm{B} 2}$ assumes the highest values. In other words, the strain in the B2 phase lattice becomes the least when partial B2 $\rightarrow$ B19' martensite transformation occurs in the layer presumably due to partial stress relaxation in the main B2 phase. According to the X-ray data, the thickness of the layer in which the relaxation processes involved in the $\mathrm{B} 2 \rightarrow \mathrm{B} 19^{\prime}$ martensite transformation are observed is $10-15 \mu \mathrm{m}$. It should be noted that despite the considerable decrease in the intensity of all diffraction peaks in the asymmetric Bragg diffraction geometry, no B19' peak is found on the corresponding diffraction patterns of the TiNi specimens after electron beam treatment. This means that

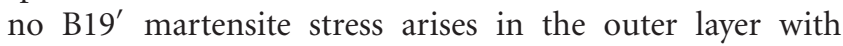
submicrocrystalline B2 structure.

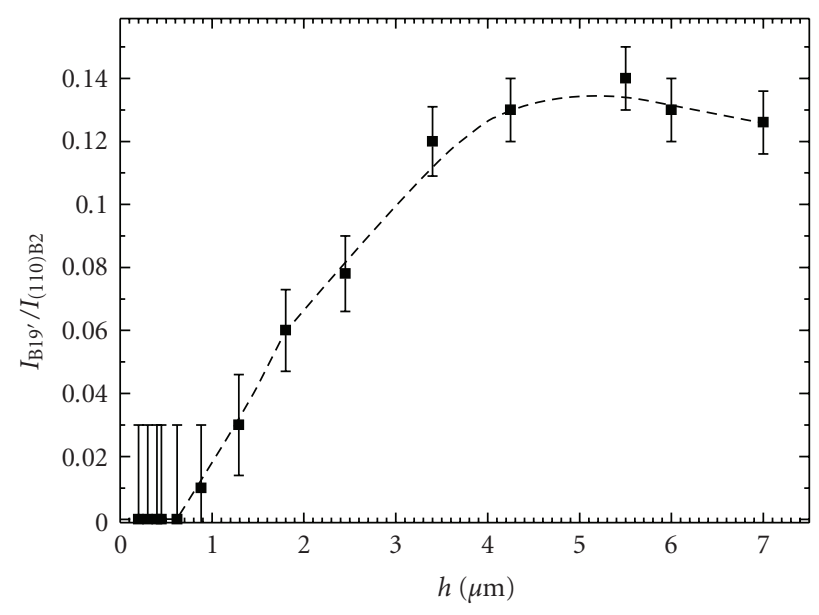

FIGURE 7: Ratio of the total intensity of the $\mathrm{B} 19^{\prime}$ peaks to the intensity of the $(110)_{\mathrm{B} 2}$ peak $\left(I_{\mathrm{B} 19} / I_{(110) \mathrm{B} 2} \sim v_{\mathrm{B} 19^{\prime}} / \nu_{\mathrm{B} 2}\right)$ versus the penetration depth in the TiNi specimens immediately after electron beam treatment.

\section{Conclusion}

The results of study show that low-energy high-current electron beam treatment of the TiNi specimens in the pulsed melting mode results in a mixed $(2 \mathrm{D}$ columnar and $3 \mathrm{D}$ equiaxial) submicrocrystalline structure on the irradiated side of the specimens. The volume fraction of each structure type in the layer annealed form the melt depends on the beam energy parameters and on the number of pulses. Increasing the density of the electron beam energy and the number of pulses suppresses the directed (to the free surface) growth of grains and hence decreases (to the point of disappearance) the volume fraction of the $2 \mathrm{D}$ substructure.

The submicrocrystalline layer is characterized by a onephase atomic crystalline state with an ordered B2 structure formed in a molten surface layer of thickness $2-5 \mu \mathrm{m}$ on fast cooling. The formed B2 structure differs from the initial one by the much smaller lattice parameter $\left(a_{\mathrm{B} 2} \approx\right.$ $2.9930 \pm 0.0005 \AA$ and $a_{0}=3.0125 \pm 0.0005 \AA$, resp.). This is attributable to the absence of impurity atoms (oxygen, carbon, etc. [17]) in the recrystallized layer and, probably, of structural defects, which is the subject for a separate study.

The B2 phase synthesized in the layer is in the elastic stress state and is characterized by lattice microstrain due to stresses of the first and second kind $\left(\varepsilon^{\mathrm{I}} \approx \pm 1 \%, \varepsilon^{\mathrm{II}}=\right.$ $0.25 \%)$. The large microstrain component $\varepsilon^{\mathrm{I}}$ suggests that in the mixed structure, the layer with a $2 \mathrm{D}$ columnar structure is an internal stress concentrator for underlying layers of the material.

It is found that in the intermediate layer beneath the outer submicrocrystalline layer, relaxation of the irradiationinduced internal stresses takes place. It is shown that the main mechanism of the relaxation is partial B2 $\rightarrow$ B19' martensite transformation. The B19' martensite phase present in the intermediate layer leads to a decrease in lattice microstrain in the adjacent B2 phase, and the larger the volume fraction of the martensite phase in the layer, the more 
pronounced the effect. The thickness of the layer in which the relaxation processes develop through the $\mathrm{B} 2 \rightarrow{\mathrm{B} 19^{\prime}}^{\prime}$ martensite transformation is $10-15 \mu \mathrm{m}$.

\section{Acknowledgments}

The authors are thankful to Professor D. I. Proskurovsky and Professor V. P. Rotshtein from the Institute of High Current Electronic for collaboration and realization of electron beam treatment of the TiNi specimens. The work was supported under projects of SB RAS no. 3.6.2.1 and no. 57, State Contract no. 02.523.11.3007.

\section{References}

[1] D. M. Follstaedt, "Metallurgy and microstructures of pulse melting alloys," in Laser and Electron Beam Interactions with Solids, B. R. Appleton and G. K. Celler, Eds., pp. 377-388, North-Holland, New York, NY, USA, 1982.

[2] L. E. Rehn, S. T. Picraux, and H. Wiedersich, Eds., Surface Alloying by Ion, Electron and Laser Beams, ASM, Metals Park, Ohio, USA, 1987.

[3] A. D. Pogrebnyak, "Metastable states and structural phase changes in metals and alloys exposed to high power pulsed ion beams," Physica Status Solidi (A), vol. 117, no. 1, pp. 17-51, 1990.

[4] V. I. Boiko, A. N. Valyaev, and A. D. Pogrebnyak, "Metal modification by high-power pulsed particle beams," PhysicsUspekhi, vol. 42, no. 11, pp. 1139-1166, 1999.

[5] B. B. Tang, I. P. Jones, W. S. Lai, and D. J. Bacon, "Sputteringinduced nanometre hole formation in $\mathrm{Ni}_{3} \mathrm{Al}$ under intense electron beam irradiation," Philosophical Magazine, vol. 85, no. 17, pp. 1805-1817, 2005.

[6] J. Zou, K. Zhang, C. Dong, Y. Qin, S. Hao, and T. Grosdidier, "Selective surface purification via crater eruption under pulsed electron beam irradiation," Applied Physics Letters, vol. 89, no. 4, Article ID 041913, 3 pages, 2006.

[7] V. F. Nolfi, Ed., Radiation-Induced Phase Transformations, Metallurgia, Chelyabinsk, Russia, 1989.

[8] A. I. Lotkov, S. G. Psakhie, L. L. Meisner, et al., "Surface nanoengineering," in Formation of Nonequilibrium States by Electron-Ion Plasma Technologies, N. Z. Lyakhov and S. G. Psakhie, Eds., p. 276, Publishing House of SB RAS, Novosibirsk, Russia, 2008.

[9] J. F. Ivanov, V. I. Itin, S. V. Lykov, et al., "Structural-phase transformations in steel 45 exposed to a low-energy highcurrent electron beam," Izvestia RAS, Metally, no. 3, pp. 130140, 1993.

[10] D. I. Proskurovsky, V. P. Rotshtein, G. E. Ozur, Yu. F. Ivanov, and A. B. Markov, "Physical foundations for surface treatment of materials with low energy, high current electron beams," Surface and Coatings Technology, vol. 125, no. 1-3, pp. 49-56, 2000.

[11] D. Mukherjee, A. A. Khan, M. R. Kumar, et al., "Metastable surface modification processes for corrosion and oxidation control," Surface Engineering, vol. 22, no. 5, pp. 411-416, 2006.

[12] J. Zou, T. Grosdidier, K. Zhang, and C. Dong, "Mechanisms of nanostructure and metastable phase formations in the surface melted layers of a HCPEB-treated D2 steel," Acta Materialia, vol. 54, no. 20, pp. 5409-5419, 2006.

[13] Q. F. Guan, P. L. Yang, H. Zou, and G. T. Zou, "Nanocrystalline and amorphous surface structure of $0.45 \% \mathrm{C}$ steel produced by high current pulsed electron beam," Journal of Materials Science, vol. 41, no. 2, pp. 479-483, 2006.

[14] A. V. Belyi, E. M. Makushok, and I. L. Pobol, Surface Hardening with the Use of Concentrated Energy Fluxes, Nauka i Technika, Minsk, Russia, 1990.

[15] D. I. Proskurovsky, V. P. Rotshtein, G. E. Ozur, et al., "Pulsed electron-beam technology for surface modification of metallic materials," Journal of Vacuum Science and Technology A, vol. 16, no. 4, pp. 2480-2488, 1998.

[16] A. I. Lotkov, L. L. Meisner, and V. N. Grishkov, "Alloys based on titanium nickelide: ion-beam, plasma and chemical surface modification," Fiz. Metal Metalloved, no. 2, pp. 1-13, 2005.

[17] K. M. Zhang, D. Z. Yang, J. X. Zou, T. Grosdidier, and C. Dong, "Improved in vitro corrosion resistance of a NiTi alloy by high current pulsed electron beam treatment," Surface and Coatings Technology, vol. 201, no. 6, pp. 3096-3102, 2006.

[18] L. L. Meisner, A. I. Lotkov, V. P. Sivokha, V. P. Rotshtein, E. G. Barmina, and Yu. L. Girjakova, "Structural-phase condition, unelastic and plastic behavior and nanohardness of the TiNi surface layers modified by an ion- and electron irradiation," Materials Science and Engineering A, vol. 438-440, pp. 558562, 2006.

[19] L. L. Meisner, I. V. Nikonova, A. I. Lotkov, V. V. Razdorsky, and M. V. Kotenko, "Effect of ion and electron beam surface modification on the corrosion properties and biocompatibility of titanium nickelide in in vivo experiments," Perspectivnye Materialy, no. 3, pp. 1-13, 2008.

[20] V. I. Domrachev, L. A. Monasevich, V. V. Myasnikov, and Yu. I. Paskal, "Initiation of martensite transformation in titanium nickelide by a shock wave on pulsed electron irradiation," Doklady of the USSR Academy of Sciences, no. 1, pp. 79-81, 1989.

[21] J. X. Zou, T. Grosdidier, K. M. Zhang, and C. Dong, "Crosssectional analysis of the graded microstructure in an AISI D2-steel treated with low energy high-current pulsed electron beam," Applied Surface Science, vol. 255, no. 9, pp. 4758-4764, 2009.

[22] Q. Guan, Q. Zhang, and C. Dong, "Physical model of stress and deformation microstructures in AISI 304L austenitic stainless steel induced by high-current pulsed electron beam surface irradiation," ISIJ International, vol. 48, no. 2, pp. 235239, 2008.

[23] J. X. Zou, K. M. Zhang, T. Grosdidier, et al., "Orientationdependent deformation on $316 \mathrm{~L}$ stainless steel induced by high-current pulsed electron beam irradiation," Materials Science and Engineering A, vol. 483-484, no. 1-2, pp. 302-305, 2008.

[24] U. C. Archiopoli, N. Mingolo, and N. Mingolo, "Generation of hardened steel surfaces with adjustable roughness by means of a pulsed electron beam," Surface and Coatings Technology, vol. 202, no. 24, pp. 5982-5990, 2008.

[25] O. I. Nalesnik, Yu. F. Yasenchuk, and N. A. Mazurkina, "Effect of electropolishing and nitrogen ion implantation on electrochemical behavior of titanium and titanium nickelide in $\mathrm{HCl}$ solution," Shape-Memory Implants, no. 4, pp. 53-58, 1992.

[26] K. M. Zhang, J. X. Zou, T. Grosdidier, et al., "Mechanisms of structural evolutions associated with the high current pulsed electron beam treatment of a NiTi shape memory alloy," Journal of Vacuum Science and Technology A, vol. 25, no. 1, pp. 28-36, 2007.

[27] Yu. P. Mironov, L. L. Meisner, and A. I. Lotkov, "The structure of titanium nickelide surface layers formed by pulsed 
electron-beam melting," Technical Physics, vol. 53, no. 7, pp. 934-942, 2008.

[28] S. S. Gorelik, L. N. Rastorguev, and Yu. A. Skakov, X-Ray and Electron-Optical Analysis, Metallurgia, Moscow, Russia, 1970.

[29] H. Gleiter, "Nanostructured materials: basic concepts and microstructure," Acta Materialia, vol. 48, no. 1, pp. 1-29, 2000. 

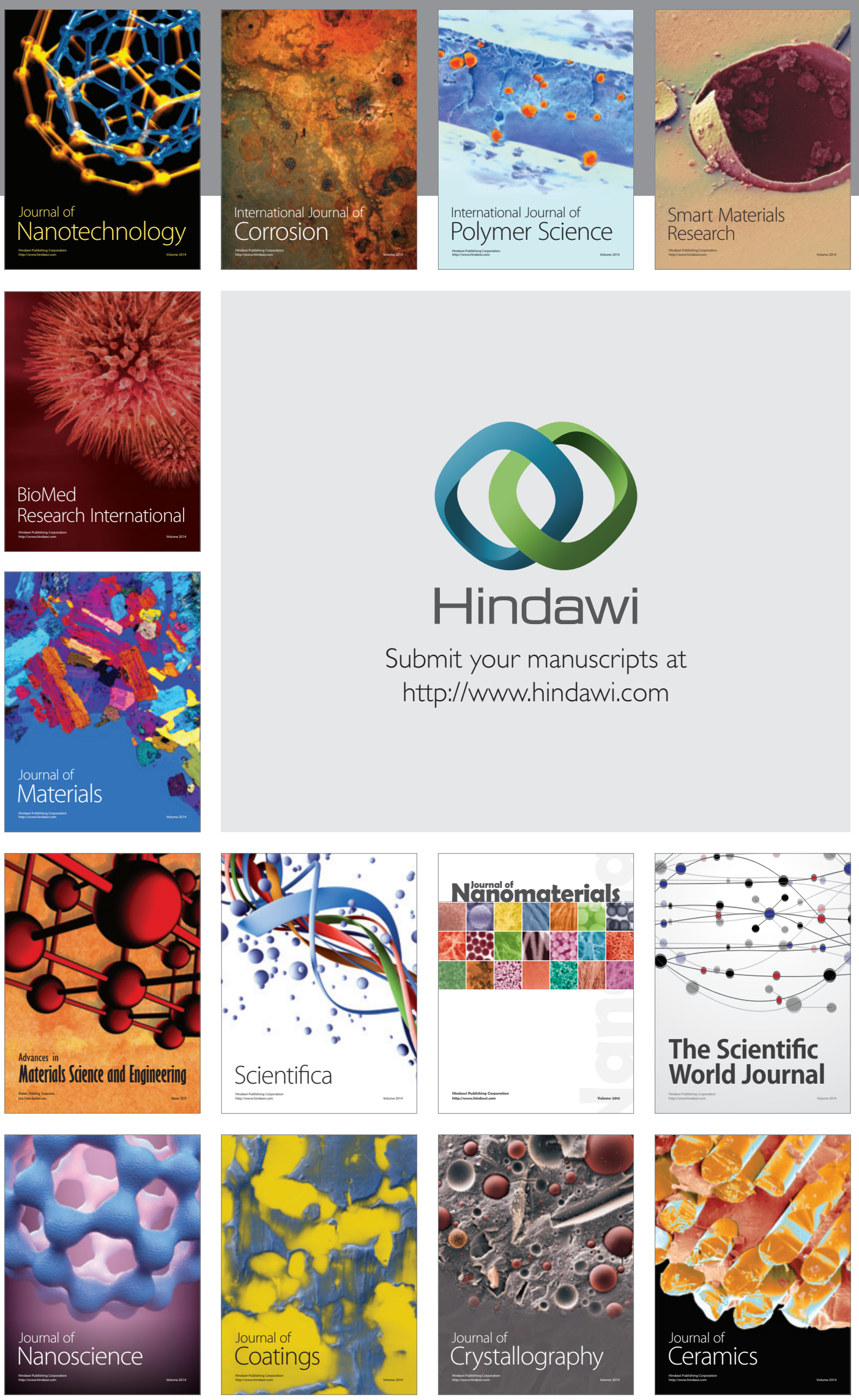

The Scientific World Journal

Submit your manuscripts at

http://www.hindawi.com

\section{World Journal}

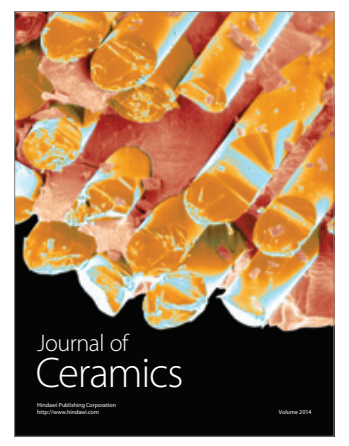

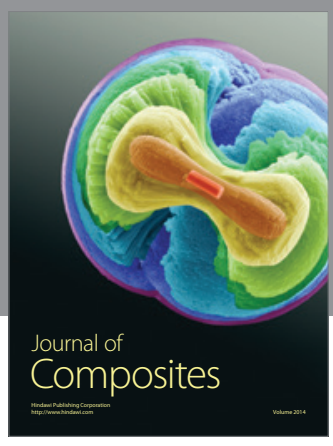
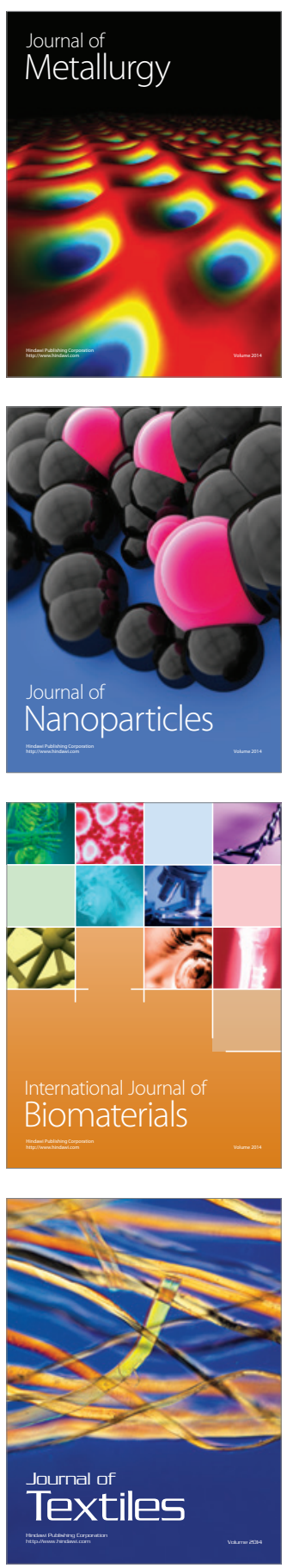